
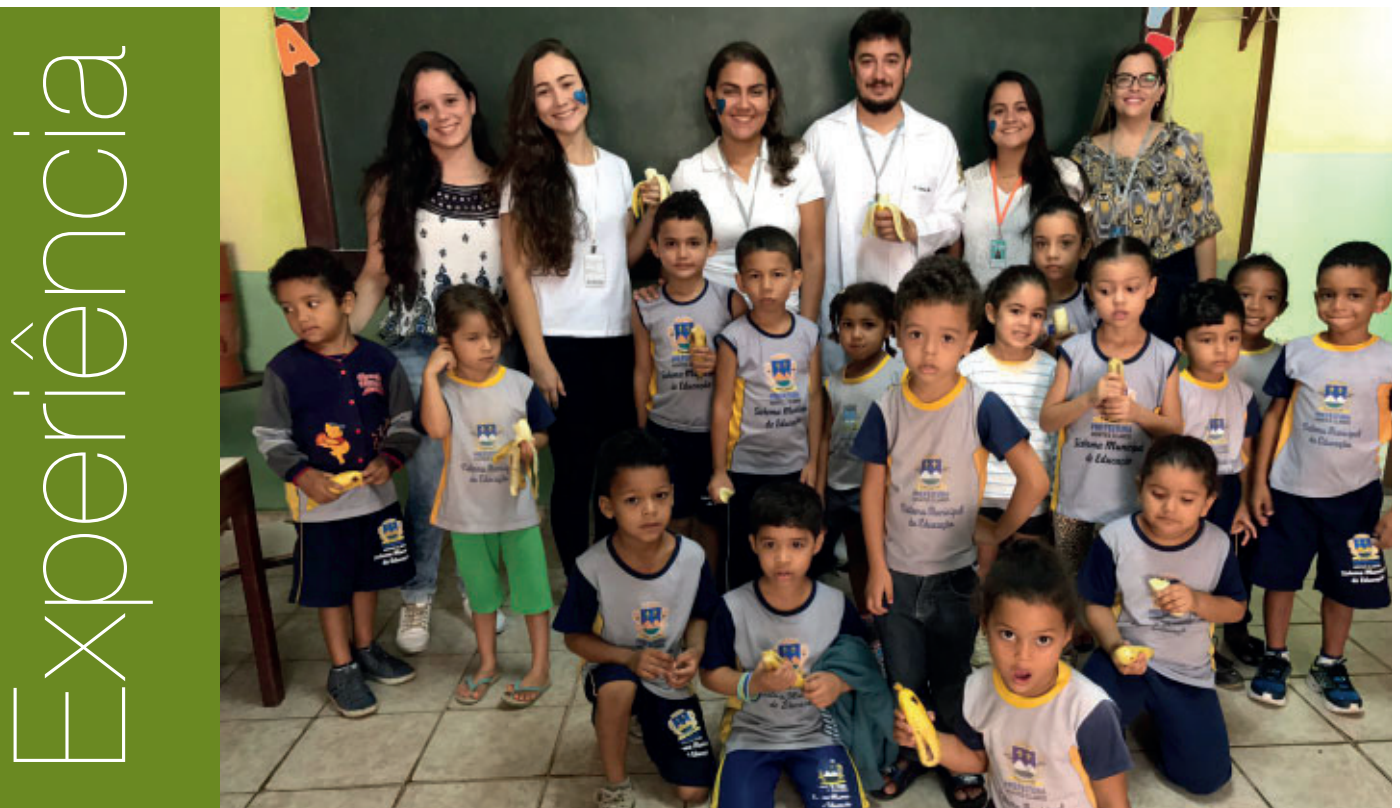

\title{
Educação em saúde: prevenção a desnutrição com crianças pré- escolares
}

\author{
Açucena dos Reis Vieira ${ }^{1}$ \\ Alice Silveira Rodrigues ${ }^{1}$ \\ Bruna Dourado Santos Loula ${ }^{1}$ \\ Maria Carolina Gomes Medrado
}

\section{RESUMO}

Trata-se de um estudo descritivo do tipo relato de experiência, realizado na creche Amiguinhos de Jesus do Bairro José Carlos de Lima de Montes Claros, Minas Gerais. Foram desenvolvidas atividades educativas lúdicas e musicais, com 16 crianças com idades entre 5 a 7 anos, para apresentar hábitos alimentares adequados em oposição a uma alimentação que ofereça problemas à saúde a longo prazo. Possui o objetivo de relatar a experiência de uma intervenção dos acadêmicos de medicina na promoção da educação em saúde, a respeito da importância de uma alimentação saudável.

\section{PALAVRAS-CHAVE}

Desnutrição. Educação em Saúde. Alimentação.

\section{ABSTRACT}

This is a descriptive study of the type of experience reported in the Amiguinhos de Jesus crèche in the José Carlos de Lima neighborhood of Montes Claros, Minas Gerais. Playful and musical educational activities were developed with 16 children aged 5 to 7 years to present adequate eating habits as opposed to a diet that offers long-term health problems. It aims to report the experience of an intervention of medical scholars in the promotion of health education, regarding the importance of healthy eating.

\section{KEYWORDS}

Malnutrition. Healt Education. Feeding.

1 Alunas de Graduação do $1^{0}$ período do curso de Medicina das Faculdades Integradas Pitágoras de Montes Claros. E-mail: alicesr8@gmail.com 


\section{Relato de experiência}

Atualmente, a educação pré-escolar tem como funções desde "guardar" crianças até preparálas para 0 ensino fundamental. Nessas instituições 0 ambiente físico, 0 grupo de atividades, as interações desenvolvidas, a rotina são essenciais para o desenvolvimento das crianças (LORDELO, 2002). Esse ambiente, no qual as crianças encontram-se em desenvolvimento, torna-se um espaço propício para a atenção profissional.

De acordo com Biscegli et al. (2006), como as crianças permanecem na creche por um longo período, elas recebem, durante este tempo, parte de suas necessidades nutricionais e é indispensável que os cuidados e a alimentação oferecidos satisfaçam seu estado nutricional e de desenvolvimento.

Nesse contexto, depreende-se que a inserção de estudantes do curso de medicina nas Equipes de Saúde da Família (ESF) tem a possibilidade de melhorar a qualidade da assistência à saúde da população do território assistido, principalmente com a atuação conjunta e integrada da equipe de saúde e dos estudantes (CAMPOS, 2007).

Assim o presente estudo objetivou relatar a experiência na promoção de conscientizar as 16 crianças com idades entre 5 a 7 anos sobre a importância de uma alimentação saudável para a prevenção da desnutrição, realizado durante 0 ensino teórico-prático da disciplina de Metodologia Científica, do $1^{0}$ período do Curso de Medicina, das Faculdades Integradas Pitágoras de Montes Claros, em uma creche Amiguinhos de Jesus, localizada no bairro José Carlos de Lima de Montes Claros, Minas Gerais; local escolhido por pertencer a área de atuação da ESF em que os acadêmicos participavam no período do $2^{\circ}$ semestre de 2016 (Figuras 1 e 2).
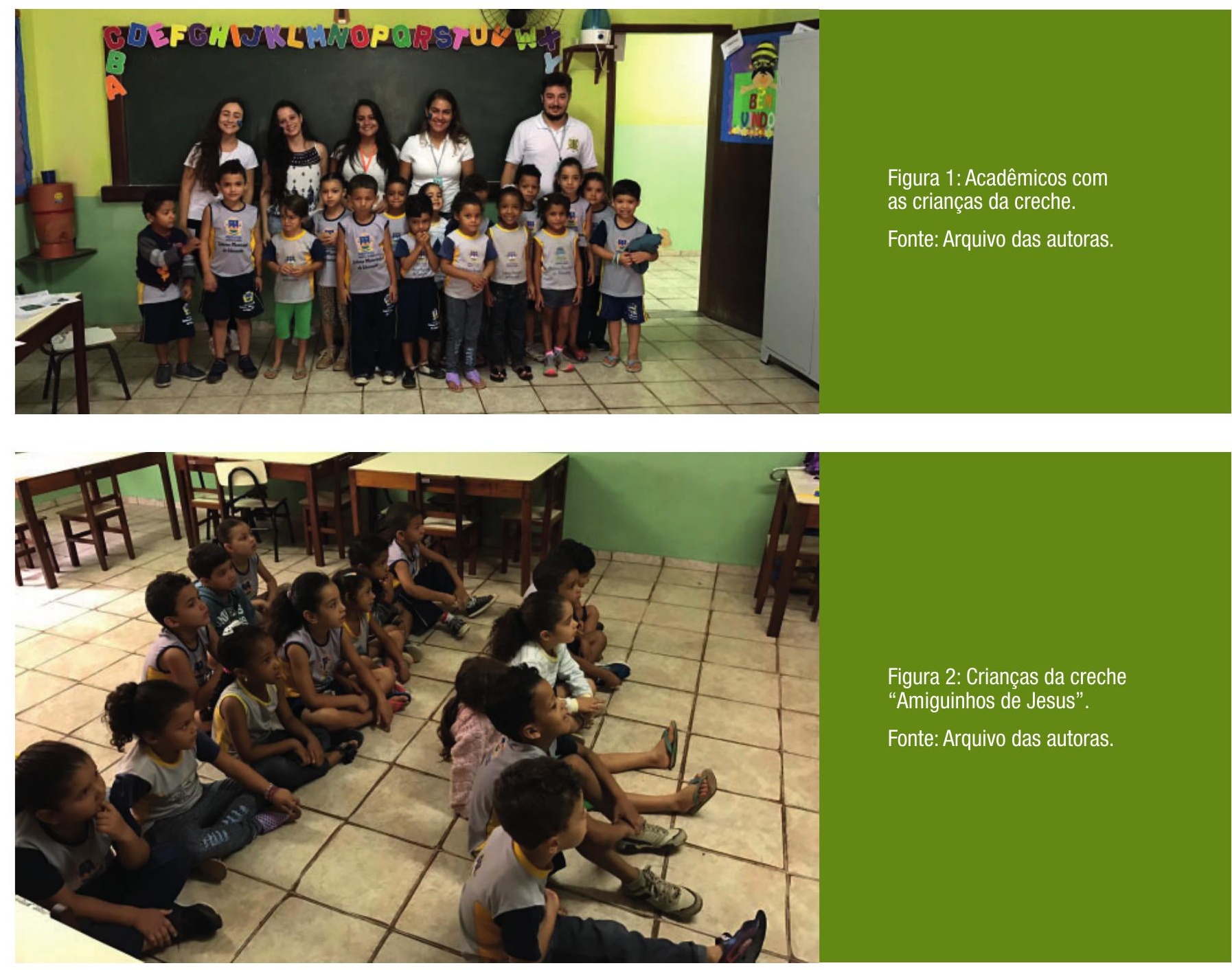
As atividades teórico-práticas desenvolvidas no campo de estágio ocorreram no período do mês de dezembro de 2016 e incluíram orientações escolares sobre a necessidade de adotarem hábitos alimentares adequados e estilo de vida saudável. Tais orientações ocorreram por meio de dinâmica realizada com a apresentação do texto "A Cesta da Dona Maricota", da autora Tatiana Belinky, e que possuía como propósito principal fazê-las indagarem-se sobre se aquilo seria ou não bons hábitos para melhorar sua qualidade de vida (Figura 3).

Figura 3: Acadêmicos e crianças da creche.

Fonte: Arquivo das autoras.

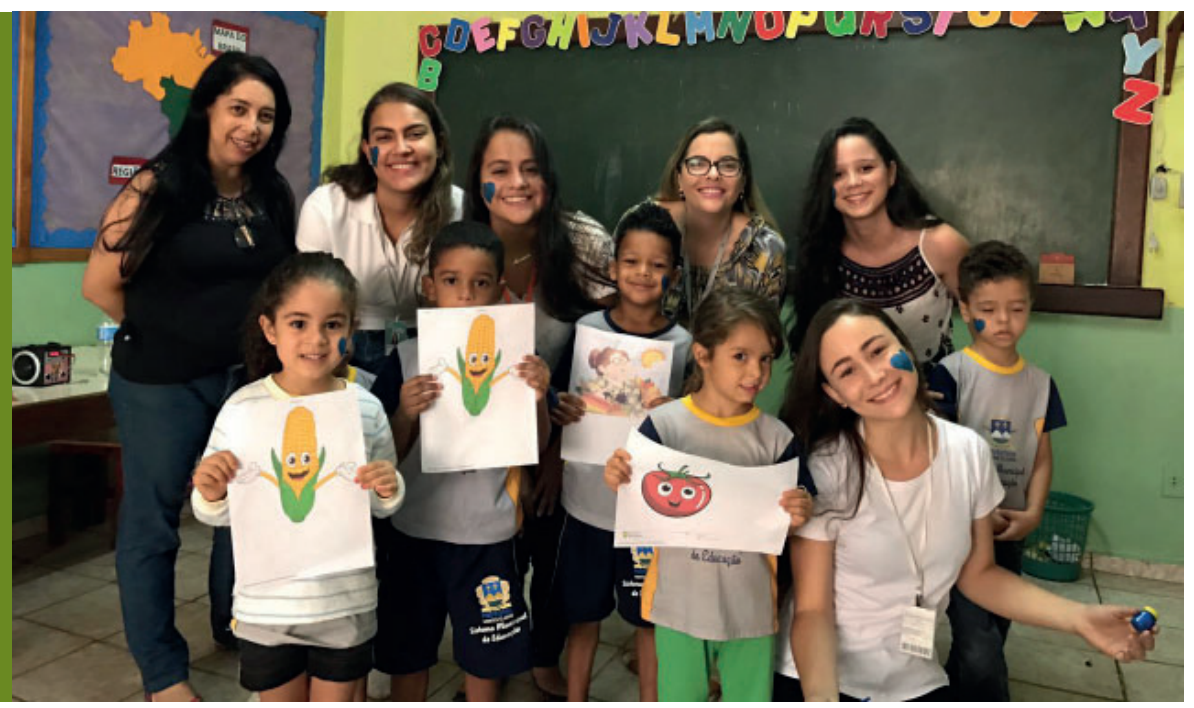

Durante a intervenção realizada pelos acadêmicos as crianças demonstraram desconhecimento acerca de alguns alimentos saudáveis, como beterraba, couve, cenoura, goiaba, abóbora, banana (Figura 4). Contudo, é de suma importância a inclusão destes, integrando uma alimentação completa e variada, para a promoção de saúde, de maneira especial, em jovens, para a prevenção doenças (B00G, 1999). Nesse sentido, uma dieta em consonância com os padrões esperados de nutrientes no período da infância é de extrema relevância para um desenvolvimento adequado, prevenindo complicações relacionadas tanto à falta quanto ao excesso de alimentos (SCOTTI, 2011).

Apesar de alguns alimentos serem notoriamente prejudiciais à saúde (refrigerante, salgadinhos e sucos industrializados, balas), estes são continuamente consumidos pelas crianças, conforme observado pelos acadêmicos. Segundo Scotti (2011) os alimentos são a fonte de nutrientes importantes e essenciais para o bom funcionamento do organismo. Logo, justifica-se a preocupação acerca da desnutrição infantil, ao se observar que a desnutrição é a segunda causa de morte mais frequente em menores de 5 anos nos países em desenvolvimento (MONTE, 2000).

Figura 4: Acadêmicos e crianças da creche.

Fonte: Arquivo das autoras.

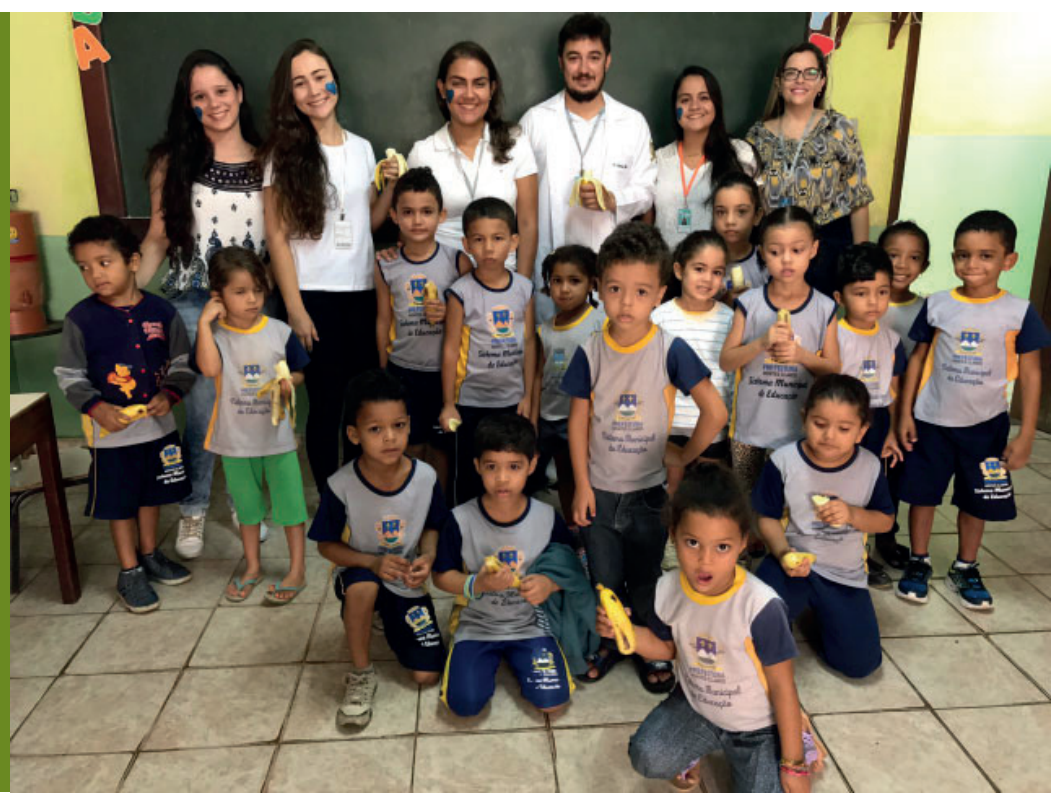


A intervenção prestada pelos acadêmicos teve como foco principal o combate à desnutrição infantil. Segundo Monte (2000) a desnutrição infantil é uma doença que pode ser desencadeada por vários fatores e possui relação com a condição socioeconômica. Ela ocorre quando 0 organismo não recebe os nutrientes necessários para o seu correto desenvolvimento, pela falta de alimentos ou má distribuição destes.

Na intervenção realizada, buscou-se, de forma lúdica, educar as crianças para prevenir a desnutrição infantil. Pôde-se notar o desconhecimento das crianças sobre quais os alimentos são saudáveis. Além disso, foi constatada a falta de instrução às crianças por parte das famílias e da equipe escolar, bem como 0 cultivo de hábitos alimentares não saudáveis. Para que o processo educativo desenvolvido pelos acadêmicos se concretize, as orientações dadas devem ser reforçadas dentro e fora da creche. Portanto, hábitos alimentares devem ser cultivados constantemente na infância.

\section{Referências}

BISCEGLI, Terezinha Soares; CORRÊA, Carlos Elysio; ROMERA, João; HERNANDEZ, Jenny Libeth. Avaliação do estado nutricional e prevalência da carência de ferro em crianças frequentadoras de uma creche. Revista Paulista de Pediatria, v. 24, n. 4, p. 323-329, 2006.

B00G, Maria Cristina Faber. Educação nutricional em serviços públicos de saúde. Cadernos de Saúde Pública, v. 15, p. S139-S147, 1999.

CAMPOS, Gastão Wagner de Sousa. Papel da Rede de Atenção Básica em Saúde na Formação Médica: diretrizes. Cadernos ABEM, Rio de Janeiro, v. 3, p. 6-10, out. 2007.

LORDELO, Eulina. Contexto e desenvolvimento humano: quadro conceitual. In.: LORDELO, Eulina; CARVALHO, Ana Maria Almeida; KOLLER, Sívia Helena. Infância brasileira e contextos de desenvolvimento. São Paulo: Casa do Psicólogo: Salvador, Bahia: Editora da Universidade Federal da Bahia, 2002. p. 5-18.

MONTE, Cristina MG. Desnutrição: um desafio secular à nutrição infantil. Jornal de Pediatria, v. 76, n. Supl 3, p. 285-97, 2000.

SCOTTI, Vera Lucia. A importância da alimentação saudável na infância. 2011. 69. Universidade Federal do Paraná, Núcleo de Educação a Distância. Cidade Gaúcha, 2011. 